\title{
Development of a Probabilistic Spark Plug Discharge Model Based on Electric Field Calculation
}

\author{
Shota Kinoshita ${ }^{\text {1) }}$ Fumiaki Aoki ${ }^{2)}$ Stefan Pischinger ${ }^{3)}$ Max Mally ${ }^{3)}$ \\ Fabian Hoppe 4) André Brunn ${ }^{4)}$ \\ 1) DENSO CORPORATION, 1-1 Showacho, Kariya, Aichi, 448-8661, Japan \\ 2) SOKEN, INC., 500-20 Minamiyama, Komenokicho, Nisshin, Aichi, 470-0111, Japan \\ 3) VKA RWTH Aachen University, Forckenbeck Str. 4, 52070 Aachen, Germany \\ 4) FEV Europe GmbH, Neuenhof Str. 181, 52078 Aachen, Germany
}

Received on July 6, 2018

\begin{abstract}
Discharge of electric field generated by a spark plug is one of the most important and influential factors to determine combustion behavior of SI engines, as it initiates flame kernels by supplying energy to the air-fuel mixture. A discharge model is required to analyze this process with the help of numerical simulation. A new probabilistic discharge model has been developed, in which the probability of the breakdown is proportional to the intensity of the local electric field. A wide variety of discharges, such as air gap discharge, surface discharge and branching discharge like corona, can be calculated with the developed model. Consequently a detailed analysis of the process from discharge to combustion can be achieved.
\end{abstract}

KEY WORDS: heat engine, spark ignition engine, Discharge Model, Optical Flow Measurement, CFD Simulation (A1)

\section{Introduction}

Discharge of the electric field generated by a spark plug is one of the most important and influential factors to determine combustion behavior of SI engines, as it initiates flame kernels by supplying energy to the air-fuel mixture. In order to analyze the ignition process by means of CFD simulations, an appropriate ignition model is needed. Colin et al. established AKTIM $^{(1)} /$ ISSIM $^{(2)}$ for simulation of the ignition. These models can be applied for air gap discharges only. However, the shape of the discharge itself is basically only determined by the mean flow. Thus, it is difficult to express more complicated motions of discharge like restrike. Furthermore, a model for advanced ignition systems in which an air gap geometry is no longer present, like corona ignition ${ }^{(3)}$, has been developed. However, there is no model for calculation of the shape of corona discharge.

For most ignition systems, the discharge is induced by a high intensity electric field. The electric field is generated by a high voltage source with values above $10 \mathrm{kV}$. Therefore, it is quite natural to take the electric field for the calculation of discharge into account. However, much time effort is required to solve the electromagnetic equations strictly from the first principle. There are some models to deal with discharge from a microscopic side. A particle based system has been developed in the field of plasma modeling, which describes plasma as individual particles (electrons, ions, and neutral molecules) ${ }^{(4)}$.

Also a probabilistic model has been developed to deal with phenomena of discharge from a macroscopic side. Niemeyer et al. established a "Dielectric Breakdown Model (DBM)" in which a probabilistic approach was adopted for the two dimensional calculation of Lichtenberg figures on the insulator ${ }^{(5)}$. The dendritic fractal shape of discharge can be simulated with this model. The idea was extended to calculate dendric breakdown patterns in solid dielectrics ${ }^{(6)}$, which is called treeing. It was as well introduced to calculate $\operatorname{lightning}^{(7)}$, which has a quite similar geometrical set up as ignition systems.

In this paper a probabilistic discharge model based on electric field calculation for the simulation of ignition as well as optical evaluations for the developed model are presented.

\section{Model Development}

\subsection{Overview}

Discharge is generally first starting from an electrode, and then growing outwards or to another electrode. The growth of the discharge is an electrical process, which proceeds in the order of nano seconds. Thus it is far below the time step resolution in computational fluid dynamics (CFD) simulations. Once discharge is generated, the discharge path is deformed by the incylinder gas flow with a much longer time scale compared to the growth of the discharge. Consequently, in the developed model these two processes are separately calculated and combined afterwards.

\subsection{Discharge growth calculation at static condition}

The growth of the discharge is calculated for stationary thermodynamic conditions in the cylinder since the growth speed is fast enough. To calculate the shape of the discharge the DBM assumption is adopted. The fundamental idea of the DBM is that 
the probability of breakdown is determined by the local intensity of the electric field. Equation (1) shows the probability of breakdown at cell $(i, j)$ on a two dimensional grid. Where $i, j, I, J$ are coordinates of a cell, $E_{i, j}$ is the intensity of local electric field at cell $(i, j)$, and $\eta$ is an index that determines the branching frequency. The calculation is performed on a square grid, and the growth of the discharge paths are calculated stepwise. The developed model has been started with two-dimensional calculation just to reveal the effectiveness of the method.

Geometrical and electrical setup of the spark plug is required for the setup of the model. There must be at least one electrode at which a voltage is applied, as well as one grounded electrode or surface as depicted in Fig. 1. Fig. 2 shows a flow chart of a sub routine for the discharge growth calculation. First of all, the electric field of each cell for the entire domain is calculated. After that the breakdown cell is determined according to the intensity of the electric field. These processes are repeated until the discharge path reaches another electrode or the length of the path exceeds a previously set maximum value.

$$
P_{B D}(i, j)=\frac{\left(E_{i, j}\right)^{\eta}}{\sum_{(\text {candidates } I, J)}\left(E_{I, J}\right)^{\eta}}
$$
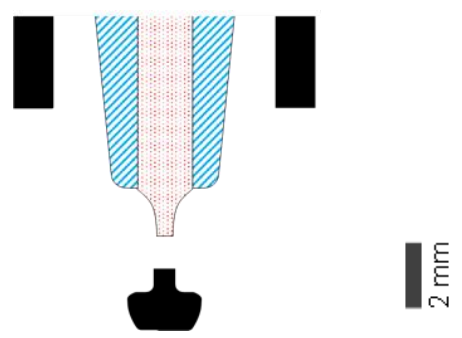

Fig. 1 Initial condition for the calculation. Solid part is metal with zero electric potential, dotted part is metal with applied voltage, and hatched part is insulator with a relative dielectric constant of 9 .

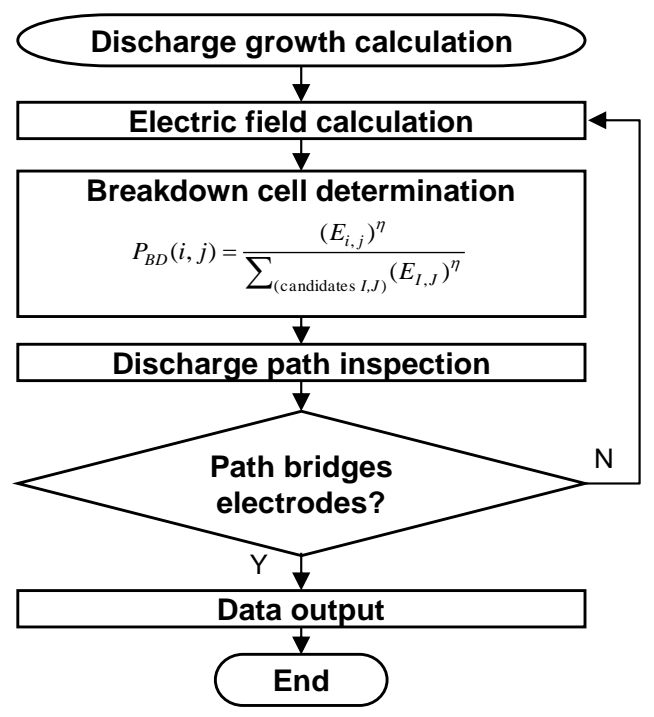

Fig. 2 Flow chart of the entire process for discharge growth calculation at static condition.

\subsubsection{Electric field calculation}

The electric potential $\phi$ is calculated by Poisson's equation shown in equation (2). $\rho_{e}$ and $\varepsilon_{e}$ are the densities of electric charge and dielectric constant. Electric field $E$ is derived from the gradient of the electric potential shown in equation (3).

$$
\begin{gathered}
\nabla^{2} \phi=-\frac{\rho_{e}}{\varepsilon_{e}} \\
E=-\nabla \phi
\end{gathered}
$$

In order to calculate $\phi$ numerically, the equation is converted into a discrete form. The simplest way is known as a finite difference method (FDM). If the medium is uniform, like for calculation of lightning in the sky or treeing in an insulator, applying FDM is quite sufficient ${ }^{(5)(6)(7)}$. However, for ignition calculation, the medium is not uniform because ceramics are used as insulation material for almost all ignition systems. Its relative dielectric constant is approximately 9 , which is very high and thus cannot be ignored. Therefore, the finite element method (FEM) based on variational calculus is utilized to calculate the electric field $^{(8)(9)}$.

Let generic function $U(\phi)$ as equation (4), Poisson's equation can be derived when the first variation of the generic function $\delta U(\phi)$ is equal to zero, as shown in equation (5). Where $\Omega$ is calculating area, $\alpha$ is an arbitrary real number, and, $v$ is an arbitrary function with a value equal to zero at the boundary of $\Omega$.. Thus $\phi$ can be calculated from $\delta U(\phi)$, which contains only first-order differentiation.

$$
\begin{aligned}
U(\phi) & =\int_{\Omega}\left\{\frac{\varepsilon_{e}}{2}(\nabla \phi \cdot \nabla \phi)+\rho_{e} \phi\right\} d V \\
\delta U(\phi) & =-\alpha \int_{\Omega}\left\{v \nabla \cdot \varepsilon_{e} \nabla \phi+\rho_{e} v\right\} d V=0 \\
& \Leftrightarrow v \nabla \cdot \varepsilon_{e} \nabla \phi+\rho_{e} v=0 \\
& \Leftrightarrow \varepsilon_{e} \nabla^{2} \phi=-\rho_{e}
\end{aligned}
$$

$U(\phi)$ for a specific cell which contains point $(i, j)$ can be calculated by the summation of adjacent four points shown in equation (6). When $\delta U(\phi)$ is equal to zero for any $\phi_{i, j}$, it yields the correct value for the electric potential. Therefore $\partial U(\phi) / \partial \phi_{i, j}=0$ for any point $(i, j)$ is required to obtain $\phi_{i, j}$.

$$
U(\phi)=\frac{1}{2} \sum_{i} \sum_{j} \int_{\Omega_{i, j}}\left\{\varepsilon_{e}(\nabla \phi \cdot \nabla \phi)+\rho_{e} \phi\right\} d V
$$

Then $U(\phi)$ at an arbitrary point $(i, j)$ can be derived with following two steps. Area 1 shown in Fig. 3 is a calculation area for point $(i, j) . U(\phi)$ is calculated from four adjacent points in this area, shown as white circles in the figure. Thus, in a first step $U(\phi)$ at $(i \pm 1 / 2, j \pm 1 / 2)$ have to be calculated. For instance $U(\phi)$ at $(i+1 / 2, j+1 / 2)$ can be calculated by dividing area 2 into four square regions and sum up all results of the integration according to equation (4). The gradient of $\phi_{i, j}$ is simply obtained from the slope of the electric potential shown in equation (7). 


$$
\nabla \phi_{i, j}=\left(\frac{\phi_{i+1, j}-\phi_{i, j}}{d}, \frac{\phi_{i, j+1}-\phi_{i, j}}{d}\right)
$$

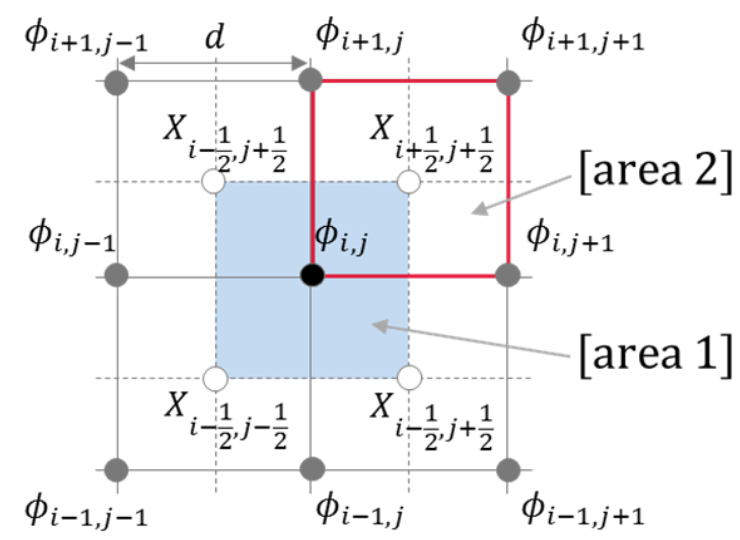

Fig. 3 Mesh structure vicinity of the point $(i, j)$ and calculating areas for each variational function.

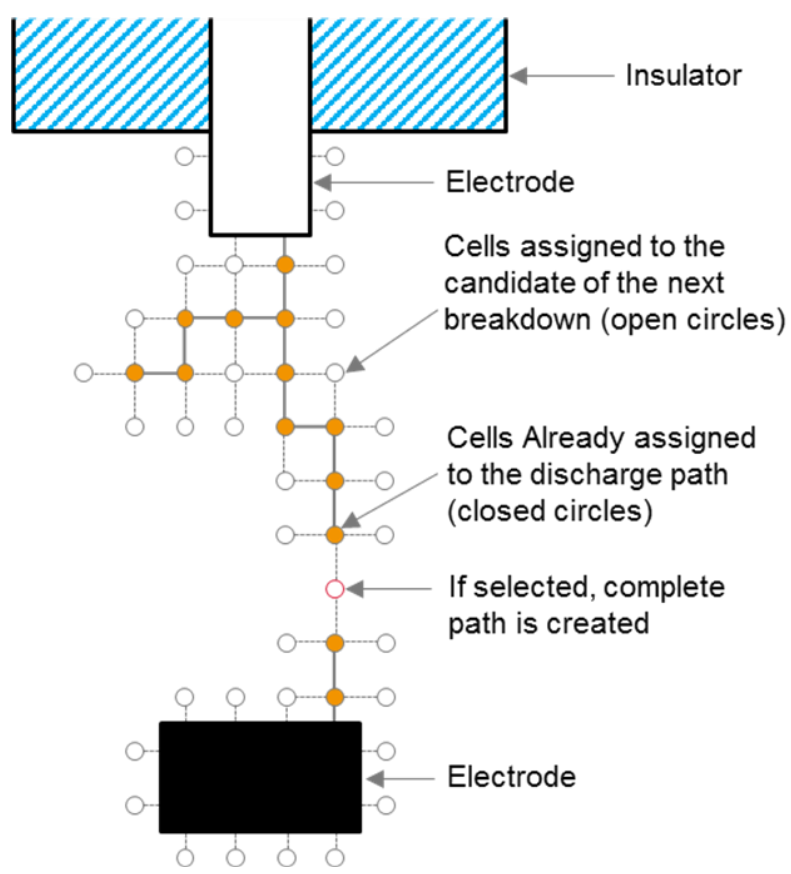

Fig. 4 Illustration of the growth of the discharge and determination of the breakdown cells. Closed circles represent cells already assigned to the discharge path, open circles represent candidate of the next breakdown, respectively.

\subsubsection{Breakdown cell determination}

Breakdown cells are stochastically determined according to the probability shown in equation (1). $\eta$ is a control parameter for the branching of the discharge. When $\eta$ is equal to one, the probability of breakdown is proportional to the local electric field which is comprehensible from the view point of physics. Hence $\eta=1$ is adopted for the calculation.

The summation in the denominator is calculated for the candidate cells that satisfy the requirements of being a breakdown cell. These requirements are as following:
1. The cell is adjacent to a cell assigned to the electrode or a cell which has already been assigned to the discharge path.

2. The cell is not assigned to the plug geometry or the discharge path.

3. The intensity of the electric field at the cell is higher than the minimum breakdown limit $E_{c}$.

Fig. 4 shows an illustration of the growth of the discharge. Due to requirement 1 , the discharge path must start from an electrode. In this case, the discharge path is calculated as closed circles in Fig. 4, open circles satisfy requirement 2 . The probability of breakdown is then calculated from the open circles, which satisfy requirement 3 as well. Finally a breakdown cell is determined according to the probability.

\subsection{Dynamic calculation of discharge deformation}

Main driving force for the deformation of the discharge is the in-cylinder flow. Therefore, the discharge model is combined with 3D-CFD. CONVERGE CFD is used for this purpose to calculate the flow field in the combustion chamber.

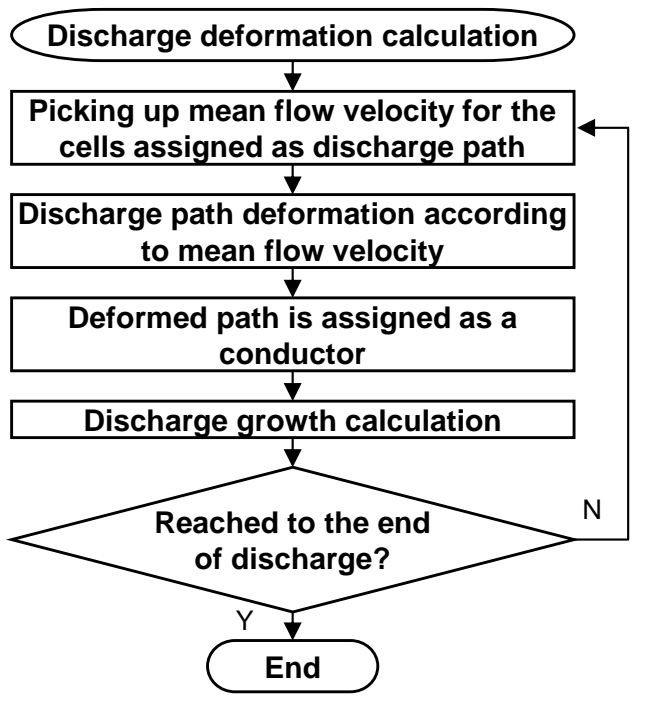

Fig. 5 Flow chart of discharge deformation sub routine.

Fig. 5 shows a flow chart of the dynamic calculation of discharge deformation. From the CFD calculation the flow velocity for the cells assigned as discharge path is picked up, and the discharge path is deformed linearly according to the velocity. Deformation is determined individually for each cell which leads to some discontinuities in the deformed path. The discontinuities are stitched up with discharge growth. The deformed path in the previous time step is assigned as a conductor in the growth calculation. Thus, the electric potential including deformed path is calculated, and then rerouting of the discharge path is achieved. In case it is easier to go directly to the counter electrode, compared to going through a deformed path due to the electric field distribution, restrike can be observed. Consequently, a more precise calculation of discharge dynamics is expected. 


\subsection{Conditions for discharge model calculation}

Two types of calculations are shown in this paper. The first one is a calculation of discharge growth under stationary conditions. These calculations proof that the developed model can be adopted to various types of discharge. The second calculation is performed considering dynamic deformation of the discharge. Flow dynamics are calculated by CONVERGE CFD with a Reynolds-averaged Navier-Stokes (RANS) approach using a k- $\varepsilon$ turbulence model. Other boundary conditions for the calculations are listed in Table 1.

Table 1 Boundary conditions for the calculations

\begin{tabular}{|l|l|}
\hline Engine speed & $20001 / \mathrm{min}$ \\
\hline Pressure of intake manifold & $1680 \mathrm{mbar} @$ valve opening \\
\hline Stroke/Bore & 1.21 \\
\hline Cylinder displacement & $400 \mathrm{~cm}^{3}$ \\
\hline Compression ratio & 13.5 \\
\hline Grid size for discharge model & $0.05 \mathrm{~mm}$ \\
\hline Applied voltage & $20 \mathrm{kV}$ \\
\hline Breakdown limit $E_{c}$ & $3 \times 10^{7} \mathrm{~V} / \mathrm{m}$ \\
\hline Branching index $\eta$ & 1 \\
\hline
\end{tabular}

\subsection{Optical measurement for verification}

In order to verify the calculation result of the model, optical measurements are performed. Particle Image Velocimetry (PIV) is applied to observe in-cylinder flow, and high speed visualization via CMOS high speed camera (Photoron SA1.1) is performed to observe the behavior of the discharge. Fig. 6 shows a scheme of the experimental setup. All measurements are performed on a motored single cylinder engine specially designed for optical measurements. A fused silica glass cylinder is adopted to provide optical access and to introduce a laser sheet. Additionally, endoscopic access through the combustion roof is prepared for observations in the vicinity of the plug. For global PIV measurements, the high speed camera is placed in position (A). As for endoscopic measurement, the camera is placed in position (B), behind the endoscope. The image distortion due to the short focal length of the endoscope and the inclined viewing direction is corrected by a reference image of a real world coordinate mesh grid in order to de-warp and scale the image data.

The PIV system comprises a double cavity diode pumped solid state Nd: YLF laser (Quantronix Darwin Duo II) operating at $527 \mathrm{~nm}$ wavelength. The interval of two laser pulses is set to 6 $\mu \mathrm{s}$. The frame synchronization between laser pulses and camera images is realized with a programmable timing unit (PTU-X) from LaVision ${ }^{\circledR}$. The in-cylinder flow is seeded with small droplets of Di-2-Ethylhexyl-Sebacat (DEHS) just upstream of the intake manifold in order to realize a homogeneous tracer distribution. The PIV data processing is performed using LaVision DaVis ${ }^{\circledR}$ 8.3.2 software.

The laser light sheet of $1 \mathrm{~mm}$ thickness is guided from the exhaust side through the glass cylinder. The visible area within the glass cylinder is limited by the light access between the nontransparent piston and cylinder head. In order to extend the visible flow area to crank angle (CA) positions close to top dead center (TDC), the piston bowl has a small slit of $3 \mathrm{~mm}$ width and $5 \mathrm{~mm}$ height. This allows illuminating the tracer close to the combustion chamber roof as well.

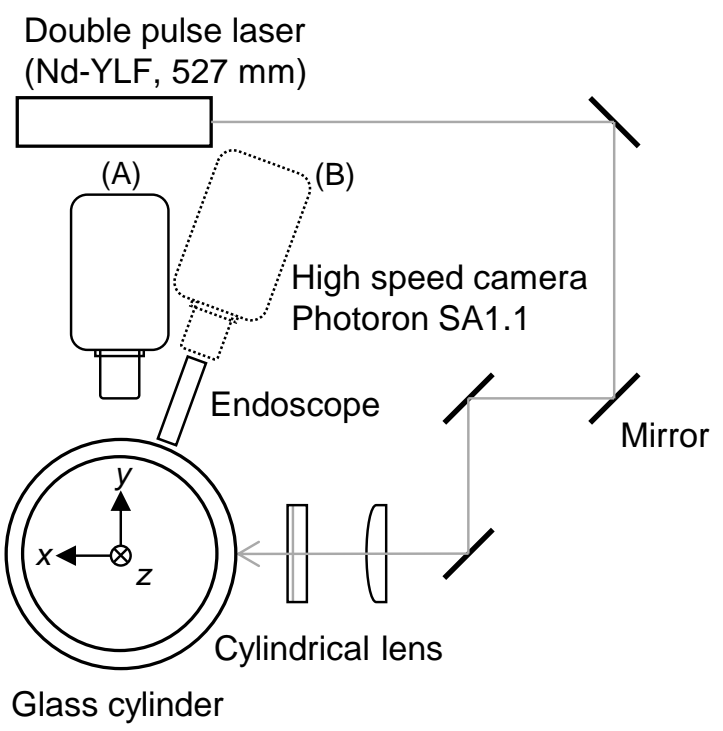

Fig. 6 Illustlation of the experimental setup for optical measurement.

\section{Result and Discussion}

\subsection{Calculation in static condition}

Fig. 7 and Fig. 8 depict the results of the calculation of discharge growth under static conditions. Three different types of ignition systems, (a) air gap system, (b) surface system and (c) corona system, are calculated. No in-cylinder gas flow is introduced, only electrical parameters are set in the calculation.

The results qualitatively agree with the real discharge. In the surface system, the discharge basically grows on the surface of the insulator, and some dendric patterns are observed ${ }^{(10)(11)}$. In the corona system, discharge starts from the tip of the electrode and grows toward the open air region. Also, some branching of the discharge path occurs ${ }^{(3)(12)(13)}$. It is shown that the principle of the discharge model, which is based on electric field calculation and probabilistic approach, works well on a wide range of discharge systems. 


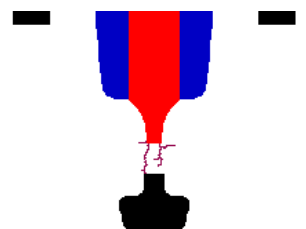

(a) air gap

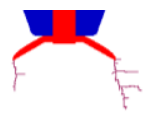

(c) corona

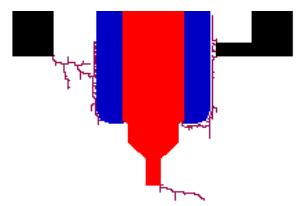

(b) surface
Fig. 7 Calculation result in static condition for three different types of ignition systems.
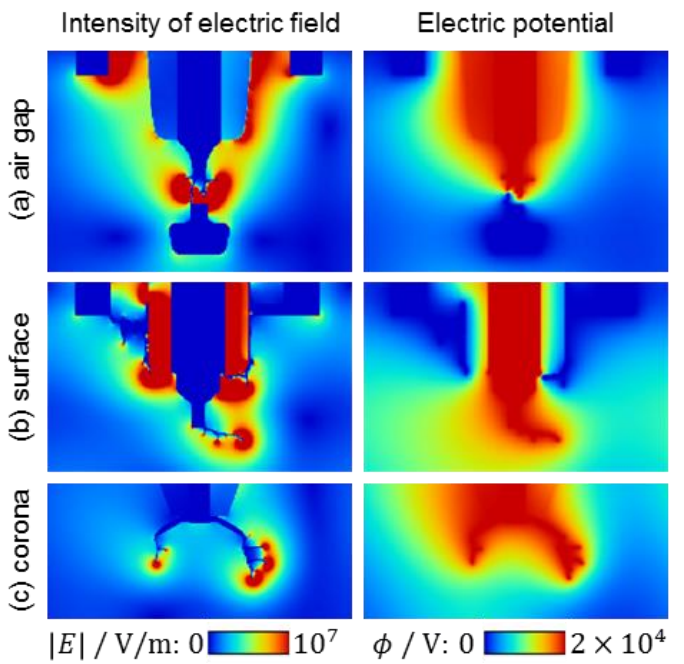

$|E| / \mathrm{V} / \mathrm{m}:$

Fig. 8 Calculation results of electrical parameters.

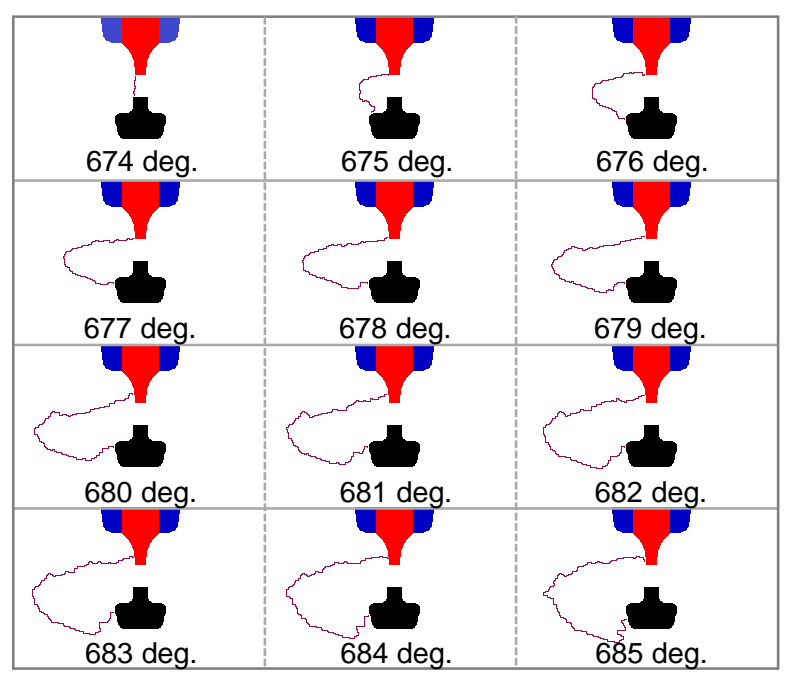

Fig. 9 Calculation result for the dynamic deformation of the air-gap discharge.

\subsection{Dynamic calculation on air-gap discharge}

Fig. 9 shows a calculation result with dynamic deformation of the discharge. The CFD results are used as a boundary condition for the depiction of the flow field. The dark pink colored pixels indicate the discharge path. On the one hand elongation is observed, and, on the other hand, both ends of the discharge are still connected to the electrodes. The movement of the ends of the discharge on the electrode surface is also observed in the experiments indicating a good agreement with the dynamic calculation.

\subsection{Verification of the model}

\subsubsection{Flow field calculated by CFD}

Fig. 10 shows a comparison of the tumble ratio curves with arotational axis normal to the measurement plane. It is calculated by dividing the angular velocity of the flow by the angular velocity of the crank shaft. The experimental results confirm the results of the CFD calculation with good agreement. The position of the two tumble peaks and their magnitude are close to each other. Especially during the compression stroke from around $660^{\circ} \mathrm{CA}$ on, which is a range of interest for the ignition, the agreement is very good.

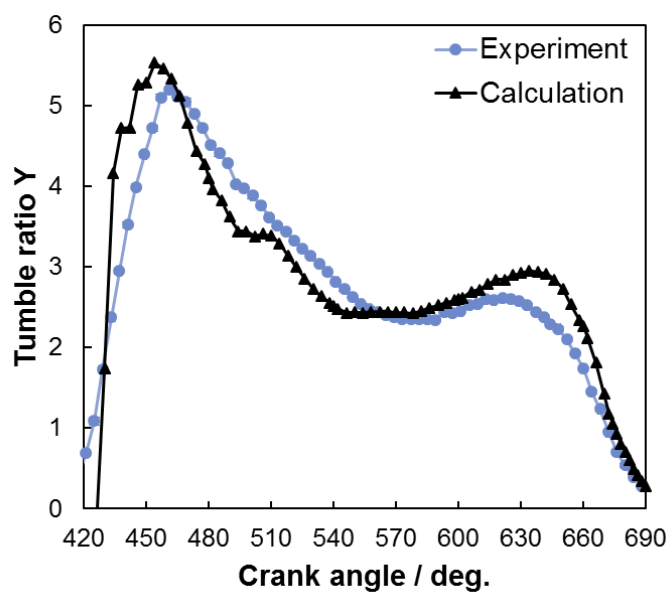

Fig. 10 Comparison of the tumble ratio $\mathrm{Y}$ derived from the experiment and CFD calculation. $\mathrm{X}$ axis show crank angle (deg.) and $\mathrm{Y}$ axis shows Tumble ratio $\mathrm{Y}$.

Fig. 11 shows actual flow distributions for, both, experiment and calculation. Absolute flow velocity $v_{a b s}=\sqrt{v_{x}^{2}+v_{z}^{2}}$ is mapped with color. The development of the shape of the tumble vortex and its position shows a similar tendency for experiment and simulation. Furthermore, absolute values of velocity are matching quite well.

Up to this point, the quality of the CFD result has been checked by global PIV measurements. With the endoscopic PIV, the flow in the vicinity of the spark plug is observed. In this case the engine load is reduced, since higher boosting pressure leads to higher peak pressure and temperature. This causes an early evaporation of the tracer particles and shortens the measurement time window close to spark timing. Therefore, intake pressure variation in global PIV is performed first to show that the difference of the charge density does not greatly influence the flow field during the compression stroke. 
Fig. 12 shows the tumble ratio for different intake pressures. During compression stroke from around $650^{\circ} \mathrm{CA}$ on, all curves are almost identical. Thus it can be concluded that the difference of the charge density has only little influence on the flow field during the compression stroke.
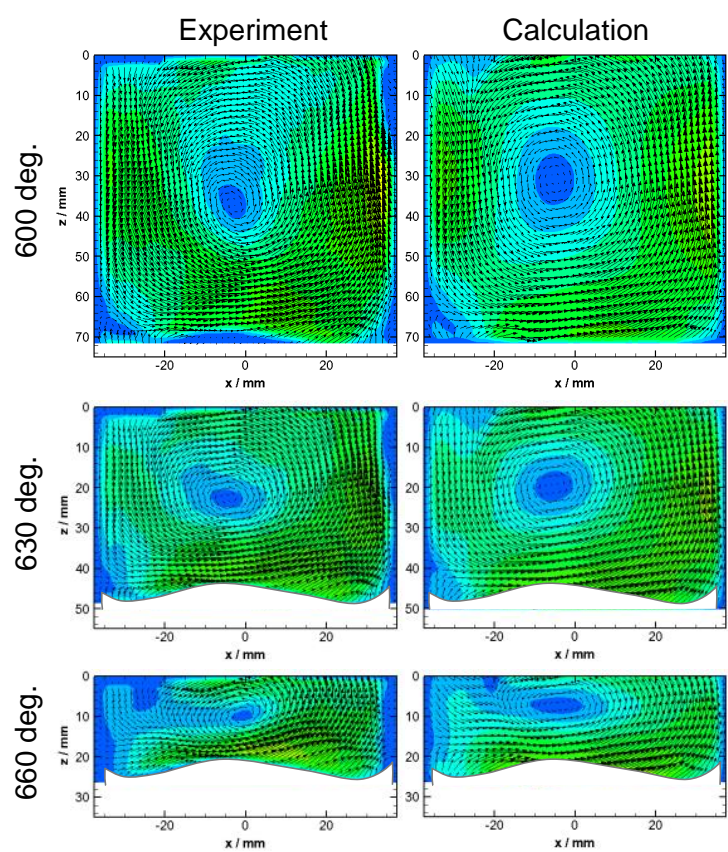

$v_{a b s} / \mathrm{m} / \mathrm{s}$

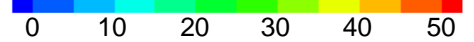

Fig. 11 Flow distribution map for the experimental result and the result of CFD calculation. Right side is intake side and left side is exhaust side.

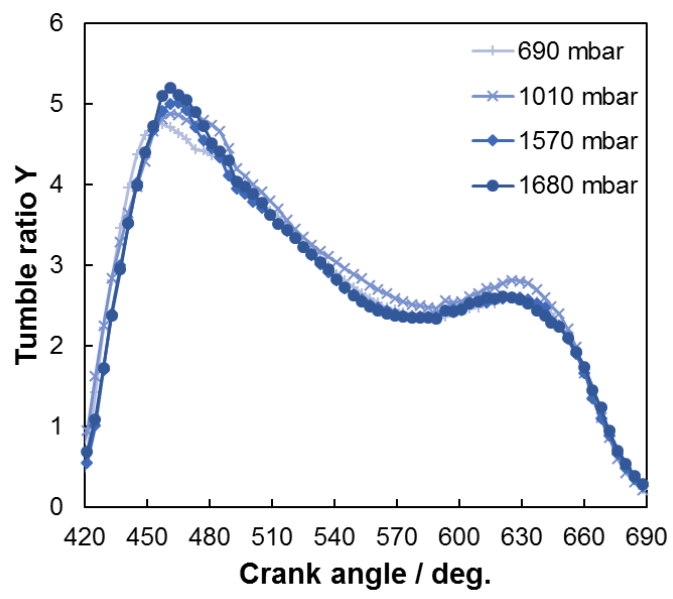

Fig. 12 Intake pressure variation of the tumble ratio $\mathrm{Y}$ in global PIV.

\subsubsection{Linear estimation of the discharge deformation}

In the developed model, the deformation of the discharge is estimated to be linearly depending on the flow. This means that the discharge completely follows the flow. In order to verify this assumption, the flow field in the vicinity of the spark plug and the motion of the discharge are analyzed with endoscopic visualizations.

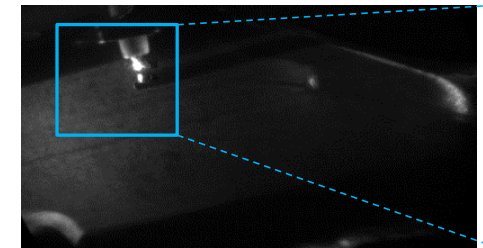

PIV (with laser \& particles)

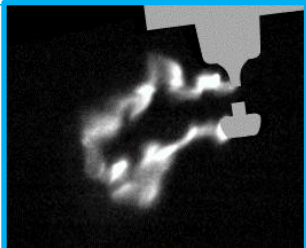

High sped visualization
Fig. 13 Exemplary images of the endoscopic measurement. Left figure shows PIV image with laser light sheet and particles. Right figure shows high speed visualization image, which is performed under higher temporal resolution compared to PIV.

Fig. 13 shows some exemplary images of this endoscopic measurement. Ignition is turned off when PIV measurement are performed. The light sheet is introduced from the left side of the figure. Due to the reflection and shading caused by the spark plug, the resolved flow velocity results just beside the plug are of limited reliability. Thus, CFD results are invoked to estimate the flow properties just beside the plug.

Resolved flow velocity calculated from the PIV measurement and the windows in which average flow is calculated are shown in Fig. 14. The size of each window is $5 \mathrm{~mm}$ square. In order to check the followability of the discharge, the averaged flow velocity in window (4) would actually be required. However, it is not available from the experiment. Therefore, first the average flow velocities in the red windows (1) to (3) are analyzed for both experiment and CFD calculation.

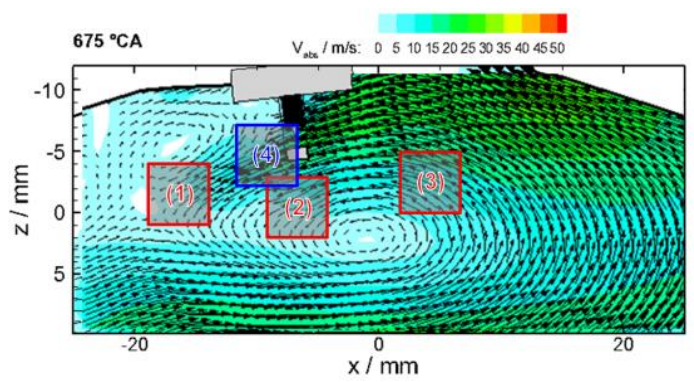

Fig. 14 Illustration of the post processed PIV result, and windows in which spatial averaged velocities are calculated. Blue window (4) mean the flow field is available only in CFD result. Right side is intake side and left side is exhaust side.

Fig. 15 shows the analyzed results for the x-component of the averaged flow velocity for, both, experiment and CFD calculation. The experimental and calculation results agree very well in window (1). Little differences are observed in window (3) due to the proximity of the window to the tumble vortex center. The flow field is very sensitive in that area. However, the tendency is well matched.

The CFD result of the averaged flow beside the plug is depicted in Fig. 16. The resulting curve in window (4) is very similar to that of window (1), especially after $675^{\circ}$ CA. Accordingly, the experimental flow velocity in window (1) is applied for the followability analysis in exhaust side vicinity of the plug instead of that in window (4). 

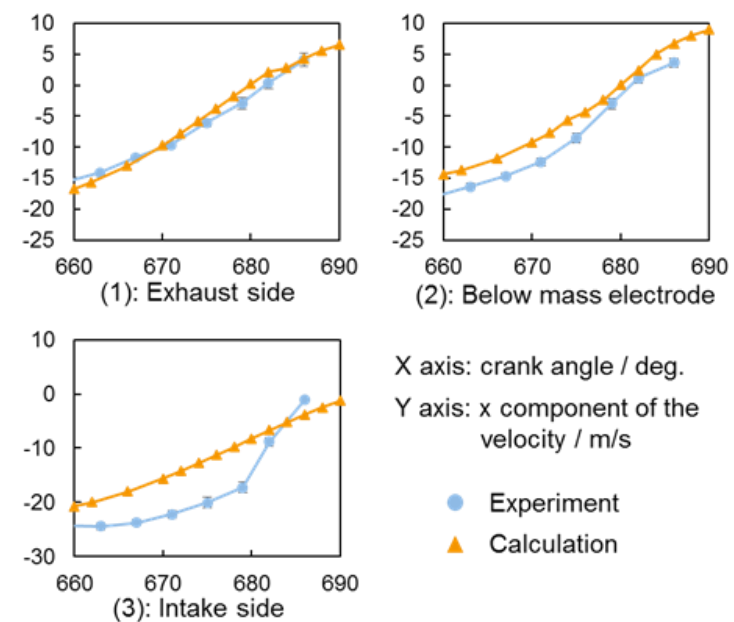

$X$ axis: crank angle / deg.

$Y$ axis: $x$ component of the velocity $/ \mathrm{m} / \mathrm{s}$

- Experiment

A Calculation

Fig. $15 \mathrm{X}$ component of the averaged flow velocity analyzed in each red windows on the experimental result.

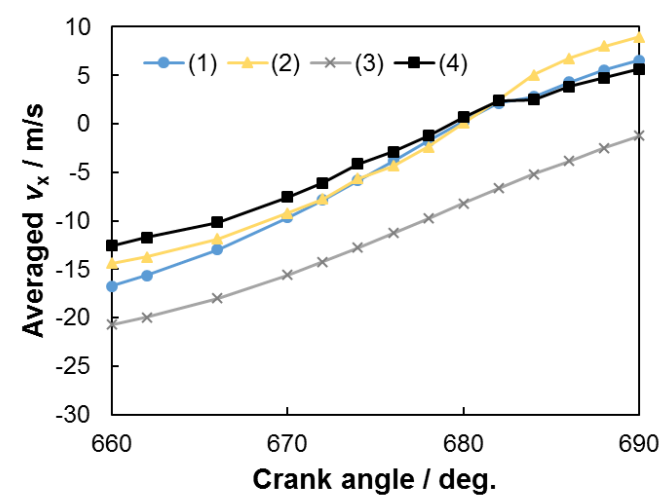

Fig. $16 \mathrm{X}$ component of the averaged flow velocity analyzed in each red and blue windows on the CFD calculation result.

The discharge movement is analyzed with the high speed visualization data. In the visualization shown on right hand side of Fig. 14, no light source is introduced. Therefore, only the light emission emitted from the discharge is observed. At the beginning of the analysis, the pictures are masked with the shape of the plug, and the combustion chamber in order to avoid that reflections are considered as discharge. In a next step, the pictures are binarized with a fixed threshold. From the binary pictures, the center of gravity of the discharge is calculated. As the center of gravity is not sensitive to the thickness of the discharge, it is adopted as a base statistic value for the evaluation of the discharge movement. The origin of the calculation for the center of gravity is set to the middle point of the air gap.

A discharge elongation velocity for $\mathrm{x}$ direction $v_{d x}$ is defined as shown in equation (8). Where $\Delta t$ is the time resolution of the measurement and $\Delta x_{c g}$ is the displacement of the center of gravity in $\mathrm{x}$-direction within the time resolution. Fig. 17 shows a comparison of the flow velocity and discharge elongation velocity. It is found that the agreement is quite good, which indicates that the discharge follows the flow field and the linear estimation method adopted in the developed model is reasonable.
Some large fluctuations are observed during the beginning of the discharge due to the instability of the breakdown process.

In the followability analysis $\mathrm{x}$-component of the flow is focused on, as it shows uniform trend in each window. Zcomponent is available in PIV as well, however, it is almost onetenth of the $\mathrm{x}$-component and the trend is not uniform. Ycomponent is almost negligible in this tumble-dominant engine.

$$
v_{d x}=\frac{\Delta x_{c g} \times 2}{\Delta t}
$$

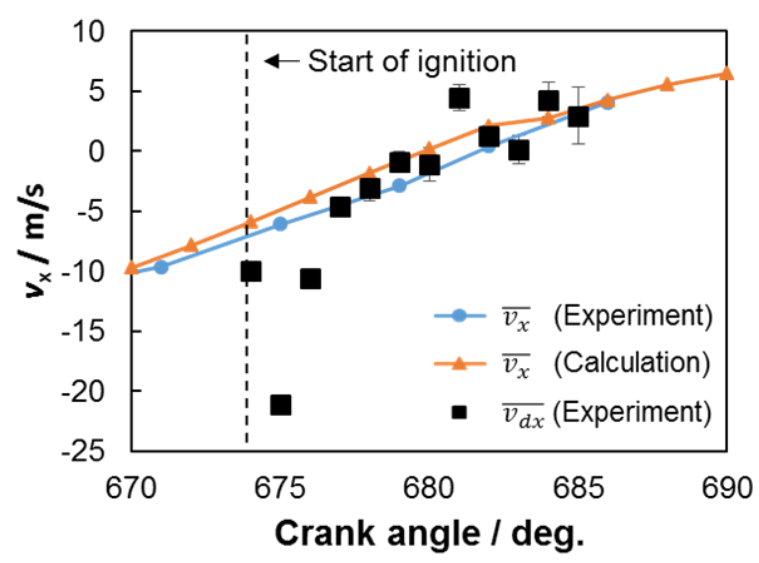

Fig. 17 Followability of the discharge movement. $v_{d x}$ indicates the velocity of the tip of the discharge.

\subsubsection{Statistical analysis of the discharge model}

Finally the result of the developed model is evaluated by the comparison of center of gravity. Fig. 18 depicts the development of the center of gravity for, both, the experimental and CFD calculation results.

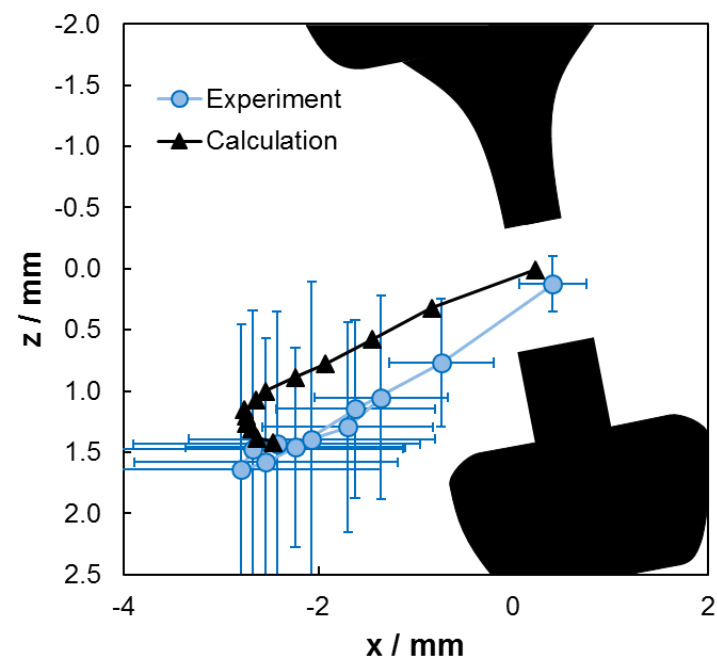

Fig. 18 Comparison of center of gravity: Experimental data is averaged for 50 cycles and error bar indicates standard deviation.

The experimental results are averaged for 50 cycles on each time step, and the error bar shows the standard deviation. The calculation result fits to the experimental scatter. The last phase 


\section{Shota Kinoshita et al. / International Journal of Automotive Engineering}

Vol.10, No.1 (2019) pp.65-72

of the elongation shows little difference since it is highly depends on a restrike of the discharge. However, the tendencies for tilt angle of the trajectory, farthest point, interval of the points during the beginning phase, and backward movement in $\mathrm{x}$ direction are very similar. Hence, the calculation results of the developed model are well capable to describe the averaged motion of the discharge.

Consequently, the approach of the developed model is reliable and proven by experiments. It is worth to be utilized as an ignition model in combustion simulation to further improve combustion analyses.

\section{Conclusion}

The discharge properties of the electric field generated by a spark plug are essential for the ignition and combustion in SI engines. Hence, a discharge model based on electric field calculation has been developed for the analysis and simulation of the ignition process. The Dielectric Breakdown Model (DBM) approach is adopted in the model in which the probability of the breakdown is proportional to the intensity of the electric field. Calculation under static conditions (not considering the gas motion in the combustion chamber) were performed for three different spark plug types showing qualitatively good agreement with experimental results.

Calculations under dynamic conditions combined with CFD simulations of the in-cylinder flow were performed as well and results could be verified by optical measurements. The deformation of the discharge plume is linearly estimated from the mean flow velocity calculated in CFD. PIV and high speed visualization techniques were used for the verification.

At first, the result of the CFD calculation with the RANS model is validated with optical PIV measurements. The flow field of the simulation well matched the experimental results and thus the flow field of the simulation is used as input for the discharge model. Then, the followability of the discharge to the flow field is evaluated. The discharge elongation velocity, defined as a twofold of the moving velocity of center of gravity, is compared to the flow velocity. It is found that the discharge elongation velocity is almost equivalent to the flow velocity. Hence it could be proven that the linear estimation method for the discharge deformation works quite well. Finally, the dynamic development of the discharge is evaluated by the comparison of the center of gravity of the discharge. The calculation result fits to the scatter of the experimental results and also shows good agreement regarding cycle averaged data. Hence, it is demonstrated that the developed probabilistic discharge model describes the real discharge with good accuracy.

The model can be applied to various ignition systems, and can be combined with flow calculations. It is worth to be utilized for the improvement of combustion simulations. The model can further be used for a detailed analysis of cycle-to-cycle variations during the ignition process in large eddy simulations.

This paper is written based on a proceeding presented at JSAE Spring Congress 2018.

\section{References}

(1) O. Colin, A. Benkenida and C. Angelberger: 3D Modeling of Mixing, Ignition and Combustion Phenomena in Highly Stratified Gasoline Engines, Oil \& Gas Science and Technology - Rev. IFP, Vol. 58, p.47-62 (2003).

(2) O. Colin, K. Truffin: A spark ignition model for large eddy simulation based on an FSD transport equation (ISSIM-LES), Proc. of the Combustion Institute, Vol. 33, Iss. 2, p. 3097-3104 (2011).

(3) Jian-Bang Liu, Paul D. Ronney and Martin A. Gundersen: Premixed flame ignition by transient plasma discharges, Proc. 3rd Joint Meeting US Sect. Combust. Inst., p. 16-19 (2003).

(4) J P Verboncoeur: Particle simulation of plasmas: review and advances, Plasma Phys. Control. Fusion, Vol. 47, No. 5A, p. 231-260 (2005).

(5) Niemeyer, L., Luciano Pietronero, and H. J. Wiesmann: Fractal dimension of dielectric breakdown, Physical Review Letters, Vol. 52, Iss. 12, p. 1033 (1984).

(6) H. J. Wiesmann and H. R. Zeller: A fractal model of dielectric breakdown and prebreakdown in solid dielectrics, Journal of applied physics, Vol. 60, Iss. 5, p. 1770-1773 (1986). (7) S. Batjargal, et al.: Visual simulation of lightning taking into account cloud growth, Computer Graphics International 2001. Proceedings. IEEE, p. 89-95 (2001).

(8) T. Nakata and N. Takahashi: The Journal of The Institute of Electrical Engineers of Japan, Vol. 100, Iss. 1, p.45-48, (1980)

(9) Gurtin, M.: Variational principles for linear initial-value problems, Quarterly of Appl. Math., Vol. 22, Iss. 3, p. 252-256 (1964).

(10) Sobota, Ana, Eddie M. van Veldhuizen, and Winfred W. Stoffels: Discharge ignition near a dielectric, IEEE Transactions on Plasma Science, Vol. 36, Iss. 4, p. 912-913 (2008).

(11) Piock, Walter F., et al.: Ignition systems for spray-guided stratified combustion, SAE International Journal of Engines Vol. 3, Iss. 1, p. 389-401 (2010).

(12) O. Toedter, et al.: Comparing Visualization of Inflammation at Transient Load Steps Comparing, International Conference on Ignition Systems for Gasoline Engines, p. 190-203. (2016).

(13) Mraihi, A, et al.: Electrical and spectroscopic analysis of mono-and multi-tip pulsed corona discharges in air at atmospheric pressure, Plasma Sources Science and Technology, Vol. 20, No. 6, 065002 (2011). 